\title{
Research on Energy Saving of Charging Based on Mathematical Modeling
}

\author{
Ziyue Cai, Weijie Li, Wenxuan Xu, Zihan Zhao \\ Nanjing Jinling High School, Nanjing, China \\ Email: caiziyue666@126.com, liweijie66666@126.com,xuwenxuan815@126.com, zhaozihan02029@126.com
}

How to cite this paper: Cai, Z.Y., Li, W.J., Xu, W.X. and Zhao, Z.H. (2020) Research on Energy Saving of Charging Based on Mathematical Modeling. Journal of Applied Mathematics and Physics, 8, 555-583. https://doi.org/10.4236/jamp.2020.83044

Received: February 1, 2020

Accepted: March 22, 2020

Published: March 25, 2020

Copyright $\odot 2020$ by author(s) and Scientific Research Publishing Inc. This work is licensed under the Creative Commons Attribution International License (CC BY 4.0).

http://creativecommons.org/licenses/by/4.0/

\begin{abstract}
This paper studies the change of electronic energy demand when people charge various kinds of electronic products (including small and large ones) in different places, as well as costs of meeting needs. By setting up a linear programming problem with the actual demand number of various charging carriers as decision variables, with the goal of the maximum cost reduction, and with the constraint of meeting needs of various customers, this paper finds out the lowest cost scheme.
\end{abstract}

\section{Keywords}

Parameter Estimation, Computer Simulation, Linear Programming

\section{Introduction}

\subsection{Background}

Living in the so called "mobile electronic world" [1], the energy consumption for charging is increasing with the popularity of the electric devices, ranging from small items (cell phones) to large items (electric vehicles) [2]. At this rate, this new type of energy consumption will rise to a considerable amount in the near future.

\subsection{Restatement of the Problem}

We are using public facilities to charge our mobile devices all the time in the current society. Some of the mobile charging services are free for use, such as the charging sockets in airports; whereas some devices are in great demand, such as charging piles for electronic vehicles. This rises a bunch of questions. Although free charging services are mostly for small electronic devices such as mobile phones and tablets, the total amount of energy consumption could be large. 
Who pays for the "free of charge" services for us? [3] Will it lead to excessive consumption of energy? If yes, we'd better find a way to get it under control. And for the charging facilities of large electronic devices, there are considerable costs for charging piles and installation. Can we establish a mathematical model and use it to find the best strategy for different situations? These are the questions we are going to discuss in this paper.

\subsection{Research Framework}

In this paper, firstly, according to different characteristics of the energy demand of small and large electronic products, a growth model of power demand is established. Secondly, according to the above model, a cost model is established to meet the power demand growth of small and large electronic products. Thirdly, four places (schools, cafes, airports, shopping centers) with obvious differences are selected to build power demand and cost generation models for small and large electronic products. Finally, a cost optimization model is established to meet the power demand of people.

\section{Model Assumptions}

To simplify the model discussion, this paper makes the following assumptions.

\section{Assumption 1}

The effect of temperature on current changes is neglected in our model. The resistance is known to change with temperature, which may result in slight change of current in the circuit. However, the impact of temperature is so small that it is neglect-able in our model.

\section{Assumption 2}

We convert all small devices to ideal mobile phones, and the small parameters stem from the differences of devices, such as the loss of efficiency due to the aging of the devices are irrelevant.

\section{Assumption 3}

All electronic vehicles are considered as ideal electronic cars, the small parameters stem from the differences of devices, such as the output frequency of the charging pile, and the aging of cars is irrelevant.

\section{Assumption 4}

The environments are defined to be ideal and have stable customer flow. For example, when we are modeling the airport, emergencies such as bad weather or the national festival are not considered.

\section{Establishment and Application of the Energy Consumption Model}

This section studies how energy consumption varies when people use small and large electronic devices, respectively. Mathematical modeling is used, and the results can be summarized to a law of energy consumption. Using computer simulation, the law of energy consumption describes the variation of demand, in 
order to find the optimal solution to meet needs of people in different circumstances.

\subsection{Problem Analysis}

There are significant differences between the small and large electronic devices that they should be considered separately when establishing the model. Generally speaking, small electronic products, namely mobile phones, tablets, computers, etc., have relatively low power rating and cheaper maintenance cost comparing with large electronic products, such as electric vehicles.

The characteristics of different environments should be taken into account as specific parameters in the mathematical modeling, in order to reflect the differences in the functions, the average lengths of time spent, in the place is different, and the rates of willingness for customers to charge their electronic devices.

\subsection{Model Establishment and Solution}

\subsubsection{Mathematical Modelling for Energy Consumption of Small Electronic Devices}

\section{- Model Establishment}

The rated power $P$ of common small electronic products, such as mobile phones, tablets, computers, etc., can be calculated through the simple relationship between electric power, voltage and current namely:

$$
P=U I
$$

By reviewing related literature [4] and fast calculation, we list the rated voltage, current, power of mainstream mobile phones, tablets, and computer brands in the market in Table 1.

The rated power of computer-like products in is basically $65 \mathrm{~W}$; the rated power of common tablets is ranging from $10-18 \mathrm{~W}$ so the average value is 14 $\mathrm{W}$; the rated power of the mobile phone is approximately $5-25 \mathrm{~W}$ and the average value is $15 \mathrm{~W}$. The tablet and the mobile phone can be classified into one

Table 1. Relevant information about mainstream brands of small electronic products.

\begin{tabular}{|c|c|c|c|c|}
\hline & & Volt/V & Current/A & Power/W \\
\hline \multirow[t]{5}{*}{ Cellphone } & Apple & 5 & $1-3.6$ & $5 w-18 w$ \\
\hline & Samsung & 9 & 2.77 & 25 \\
\hline & LG & 10 & 2.2 & 22 \\
\hline & Motor & 5 & 1 & 5 \\
\hline & iPad & 5 & $2-2.4$ & $10-12$ \\
\hline \multirow[t]{2}{*}{ Tablets } & iPad Pro 11" & 9 & 2 & 18 \\
\hline & Samsung & 9 & 1.67 & 15 \\
\hline \multirow[t]{3}{*}{ Laptop } & $\mathrm{Mac}$ & 19 & 3.25 & 65 \\
\hline & ThinkPad & 19 & 3.25 & 65 \\
\hline & $\mathrm{Hp}$ & 19 & 3.25 & 65 \\
\hline
\end{tabular}


category, namely the mobile phones below, since their average rated power are very close.

The literature [5] shows the distribution of time spent on mobile phones per day, where $p_{1}=4 \%$ of people spend $0-1$ hours, $p_{2}=20 \%$ of people spend 1 - 3 hours, $p_{3}=29 \%$ of people spend 3 - 5 hours, $p_{4}=21 \%$ of people spend 5 - 7 hours, $p_{5}=26 \%$ of people spend $7-10$ hours. Assuming that the time of people using mobile phones within each time period is uniformly distributed, then we use the mean value, that is, the midpoint of the interval as the average time spent in each period. Thus, the average time spent on mobile phone per person per day can be calculated by:

$$
T=p_{1} \times 0.5+p_{2} \times 2+p_{3} \times 4+p_{4} \times 6+p_{5} \times 8.5=5.05(\mathrm{~h})
$$

The power consumption is directly proportional to the average time of phone usage, and so is the demand for charging. There are differences in battery capacity and life for various models of mobile phones. For simplicity, we assume that the average battery life $T_{0}=4 \mathrm{~h}$, average charging time $t_{0}=2 \mathrm{~h}$, and the average charging time per phone per person per day is:

$$
t=\frac{T}{T_{0}} t_{0}=2.525(\mathrm{~h})
$$

Assuming that the number of people in a certain environment is $N$; the proportion of people with mobile phones is $\delta_{1}$; the proportion of people carrying computers is $\delta_{2}$; the rate of demand for charging a mobile phone is $\gamma_{1}$; the rate of demand for charging a computer is $\gamma_{2}$; these rates reflect how often people use their mobile phones or computers at the given site. Let $\alpha$ denote the ratio of rated power between one computer and one mobile phone, then $\alpha \approx 4.3$ according to Table 1; let $\beta$ denote the ratio of the average time of usage between one computer and one mobile phone. The computers can be converted to mobile phones by introducing parameters $\alpha$ and $\beta$. Let $P$ be the average rated power of one mobile phone, and the average daily consumption of electronic power on the given site can be expressed as:

$$
Q=\gamma_{1} \delta_{1} N t P+\gamma_{2} \alpha \beta \delta_{2} N t P=\left(\gamma_{1} \delta_{1}+\gamma_{2} \alpha \beta \delta_{2}\right) \frac{N T t_{0} P}{T_{0}}
$$

According to (3.1.4) the rate of demand for charging mobile phones $\gamma_{1}$ is inversely proportional to the average battery life of mobile phone $T_{0}$ and is in direct proportion to other variables. The longer the average time people use a mobile phone, the higher the power consumption. Among all the parameters introduced, $P$ and $T_{0}$ are the properties related to the electronic products themselves, commonly determined by the design, functions and manufacturing process from the producers. The greater parameter $P$ means higher charging efficiency; while the larger $T_{0}$ indicates the higher the battery capacity. There are lots of other factors such as the updating and replacing of small electronic products, or the variation of age group proportion of entire population that may affect our parameters. For example, as the structure of the population changes, the 
average time spent on small mobile devices with respect to the proportion of the population $\left(p_{1}, p_{2}, p_{3}, p_{4}, p_{5}\right)$ will also change accordingly, which will cause $T$ to change (3.1.2). The energy consumption for charging small electronic devices will change, and the variation is determined by the parameters in the model (3.1.4).

\section{- Prediction by Simulation and Model Accuracy Analysis}

The development of modern information technology grants small electronic products, such as mobile phones and tablets, more functions, these smaller electronic products have replaced traditional laptops to some extends, resulting in a relative decrease in the use of computers. The proportion of energy consuming by computers will decrease, along with the predictable proportional increase in the power consumed by smaller products like mobile phones. That is, the demand for the energy consumption of mobile phones is growing faster than computers.

Assuming that the unbalance energy consumption growth between laptops and smaller electronic devices will not affect all other parameters we have introduced, we may use computer simulation to predict this trend. We assign some values to the parameters for the simulation: $N=20000, \delta_{1}=0.9, \delta_{2}=0.4$, $p=15(\mathrm{~W}), t_{0}=2(\mathrm{~h}), T_{0}=4(\mathrm{~h}), \quad \alpha=4.3$. We then assume that the average time $T$ of mobile phone usage is in accordance with the sustained growth speed $v_{1}(t)$, the rate of demand $\gamma_{1}$ for electrical energy using mobile phones increases with speed $v_{2}(t)$. The proportional reduction in computer usage can be expressed by adapting decreasing rates $v_{3}(t)$ and $v_{4}(t)$ for parameters $\delta_{2}$ and $\gamma_{2}$, respectively. The $v_{1}(t), v_{2}(t), v_{3}(t), v_{4}(t)$ are real-time parameters which can be estimated and modified by big data collection. Here we assume the rate is constant by time to simplify computer simulation. Let the base rate be $v$, so that $v_{1}(t)=b_{1} v, \quad v_{2}(t)=b_{2} v, \quad v_{3}(t)=b_{3} v, \quad v_{4}(t)=b_{4} v$.

1) Assign values to parameters:

$$
b_{1}=1, \quad b_{2}=1.2, \quad b_{3}=0.8, \quad b_{4}=0.6 .
$$

Obviously the value of parameters will vary through the introduction with the growth rates: $\gamma_{1}$ will keep increasing starting from $0.6, \delta_{2}$ will decrease from 0.5 , and it will achieve a steady-state after touching 0.3 , which means it will not keep decreasing any more. $\gamma_{2}$ will decrease from 0.4 , and become stable after reaching 0.2. Figure 1 shows the variation in energy demand overtime for 30 -time steps when the base rates are set to be 0.01 and 0.03 respectively. It can be seen that power demand is gradually decreasing: (a) the base rate is 0.01 , which is relatively slow, which means of mobile phones will keep replacing computers to some extend; and (b) the base rate is 0.03 , the speed of change becomes faster, the parameters for computers have fallen to the lowest value. So (b) gives us a replacing process in longer phase, where the first 10 steps are just the same as (a). From the beginning, computers consume more power than mobile phones, and gradually some computers are replaced by mobile phones. That is, the computer usage rate is reduced, and energy consumption will naturally 


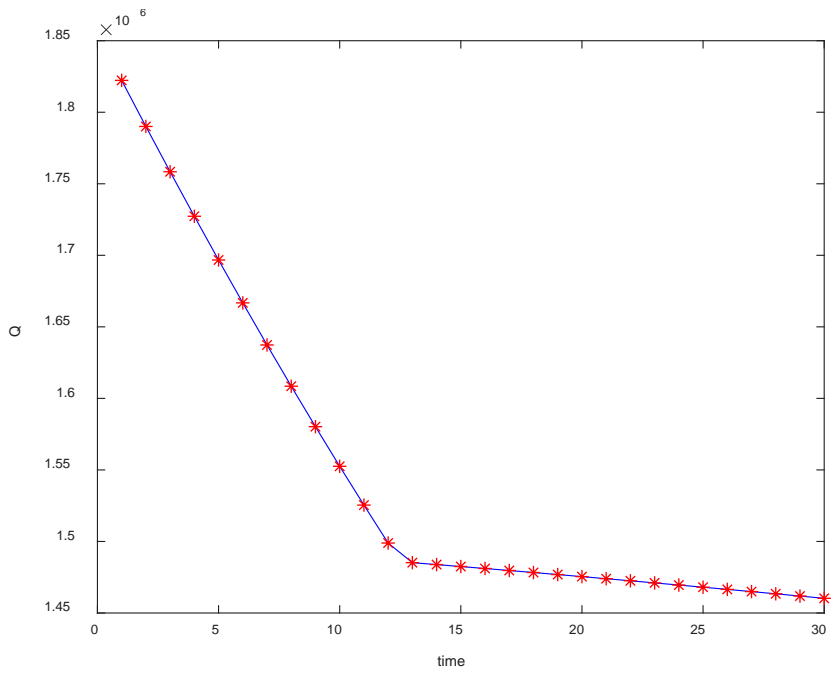

(a)

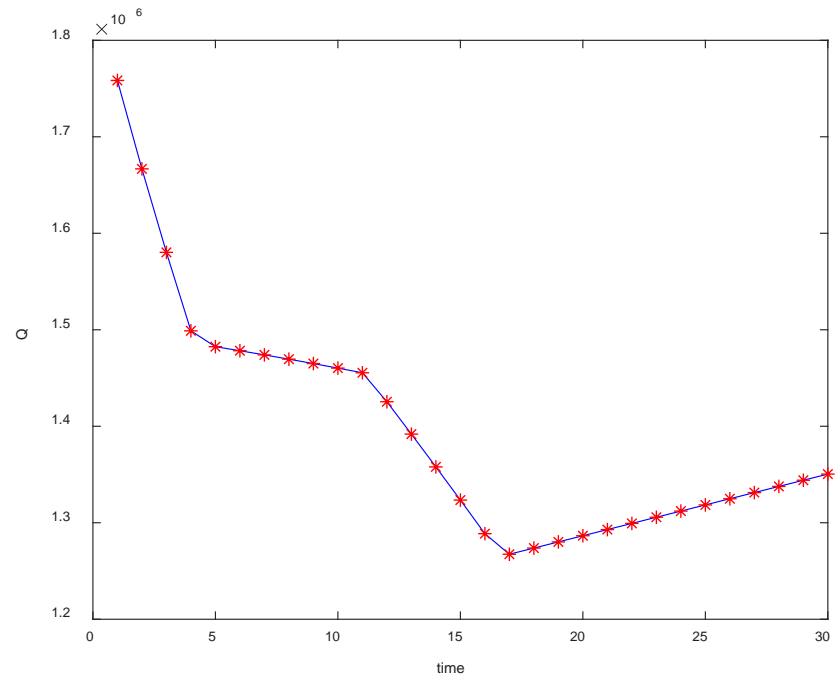

(b)

Figure 1. Power demand versus time (base rate is 0.01 and 0.03 , within 30 -time steps). (a) $\quad v=0.01$; (b) $\quad v=0.03$.

decrease. There are differences in slopes between (a) and the first 10 steps of (b) since the basic rate is different. And in (b) the slope of the curve is not the same at different stages since the variation in the growth speed of the parameters, also the time for them to reach the lower limit is different. After the computer parameters reach the lower limit, the demand for electric power will continue to increase along with the gradual increase in mobile phone usage. The result is more reasonable and close to the actual situation, which means the simulation results are reliable.

2) Assign values to parameters:

$$
v=0.03, \text { (a) } b_{3}=0.8, \quad b_{4}=0.6 \text {; (b) } b_{3}=0.4, \quad b_{4}=0.2 ;
$$

Figure 2 shows the change in power demand with respect to time when applying different modification factors to the parameters of computers, while keeping the growth rate the same for the parameters of mobile phones: $b_{3}=0.8$, $b_{4}=0.6$ for (a) and $b_{3}=0.4, b_{4}=0.2$ for (b). The two images look similar, while the duration of each phase is not the same, which is believed to be determined by the speed at which the mobile phones are replacing the computers.

3) Assign values to parameters:

$$
v=0.03, \text { (a) } b_{1}=0.8, \quad b_{2}=1 ; \text { (b) } b_{1}=1.2, \quad b_{2}=1.4 ;
$$

Figure 3 shows the change in power demand with respect to time when applying different modification factors to the parameters of computers, while keeping the growth rate the same for the parameters of mobile phones: $b_{1}=0.8$, $b_{2}=1$ for (a) and $b_{3}=0.4, b_{4}=0.2$ for (b). The two images look similar, while the duration of each phase is not the same, which is believed to be determined by the speed at which the mobile phones are replacing the computers.

Both Figure 2 and Figure 3 show us how the energy consumption changes about time at different growth rates that the larger electronic devices, represented 


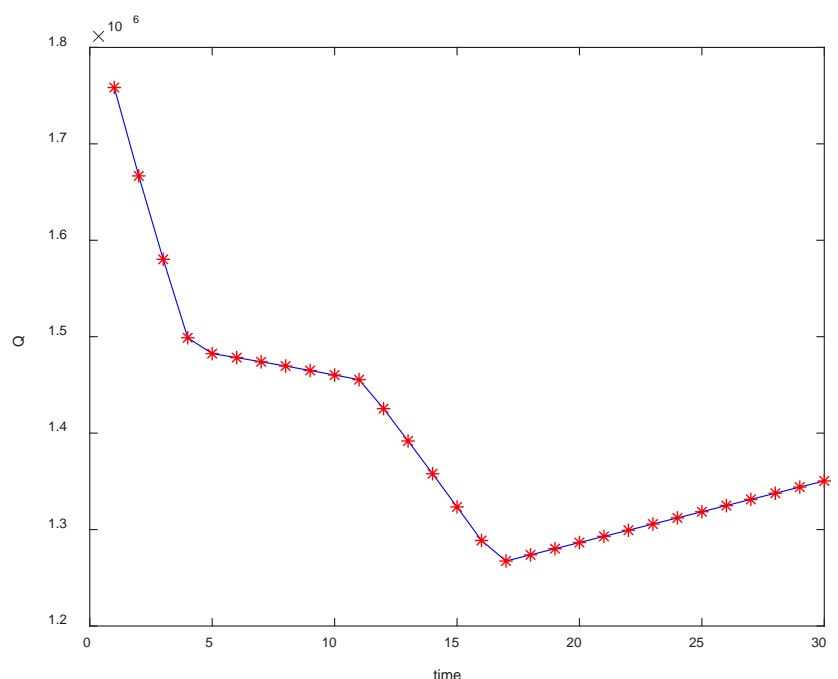

(a)

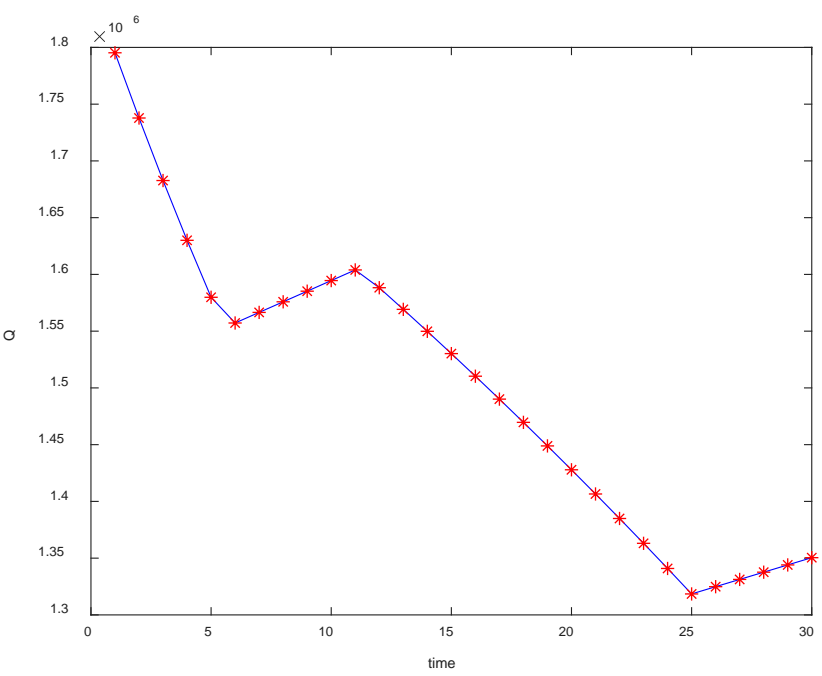

(b)

Figure 2. The change in power demand versus time (different growth rate for computes; same growth rate for phones). (a) $b_{3}=0.8, b_{4}=0.6$; (b) $b_{3}=0.4, b_{4}=0.2$.

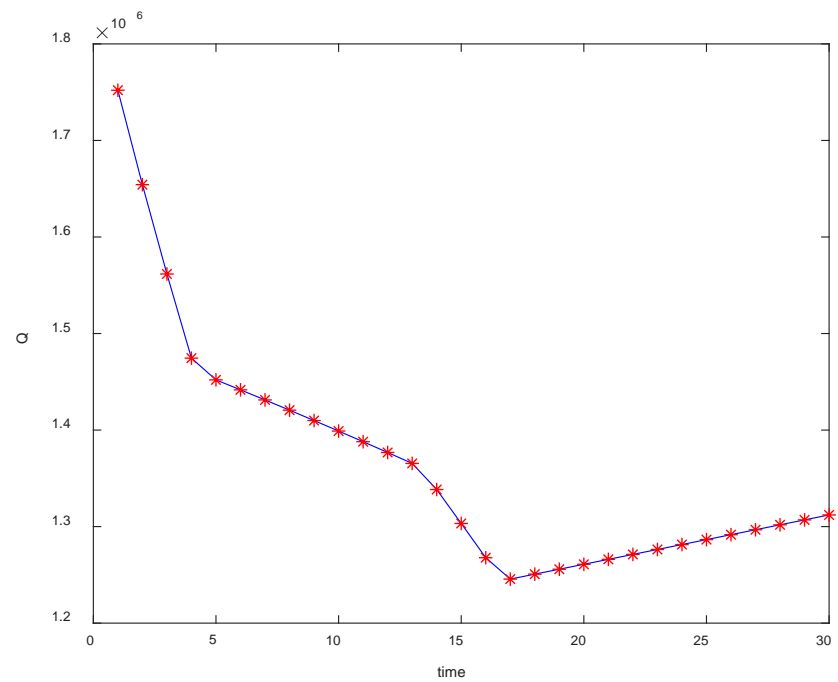

(a)

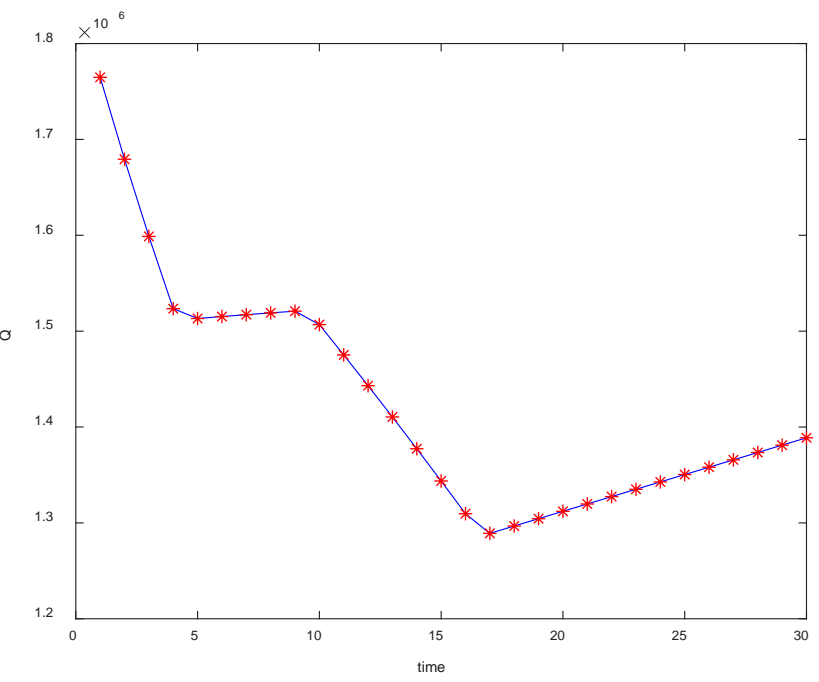

(b)

Figure 3. The change in power demand versus time (different growth rate for phones; same growth rate for computers). (a) $b_{1}=0.8, b_{2}=1$; (b) $b_{1}=1.2, \quad b_{2}=1.4$.

by computers, are replaced by the smaller electronic devices, represented by mobile phones. Generally speaking, all graphs show a common 4-phase tendency, no matter how the parameters change. But the details in the graphs such as the slop and the duration of each phase are determined by the rate of phone-replacing-computer process. In this mathematical model, the parameters can be easily adjusted and simulated by computer, the result is reasonable and easy to understand, which will give us the approximate picture of variation of energy consumption of charging small products, including phones, tablets, laptops, etc., in the future. 


\subsubsection{Mathematical Modelling for Energy Consumption of Large-Scale Electronic Products}

\section{- Model Establishment}

According to Hypothesis 2.1, as the effect in real-time power caused by temperature during the charging process has been ignored, and we will keep using the formula (3.1.1) to do the calculation for the power of large electronic products.

According to the literature [3] and simple calculation, the average power consumption per mile of an electric car is $320 \mathrm{Wh}$. Table 2 gives the basic information of three electric vehicles with high sales market share. Although the car brands are different, the maximum time required for the battery to be fully charged is close. The average time can be taken as $10 \mathrm{~h}$, which is the time that it needs to charge a common electronic car by level 2 charging piles. According to the literature [6], Chevrolet Bolt EV is not suitable for long distance travel far because its maximum range is too small. Thus it belongs to the family of small electronic vehicles. The differences between the common electronic car made by any other mainstream producers and one of the six models in our table can be neglected in all aspects that matter. Thus we can categorize all electronic cars in the market to the six types and continue our calculation with the approximation.

Assuming that the six models above have a market share of $\lambda_{1}, \cdots, \lambda_{6}$ respectively and $\lambda_{1}+\cdots+\lambda_{6}=1$, then the average maximum range of the electric vehicle can be obtained by:

$$
L=\lambda_{1} \times 156+\lambda_{2} \times 234+\lambda_{3} \times 125+\lambda_{4} \times 187+\lambda_{5} \times 265+\lambda_{6} \times 45
$$

Table 3 provides information about common charging piles. For simplification, we use $22 \mathrm{~kW}$ as our base rated output power the parameter a denote the ratio between the other charging piles and the basic, thus the charging time is $T_{\text {car }}=10 / a$ in our model.

Table 2. The basic data for three popular electronic vehicle models.

\begin{tabular}{ccccc}
\hline Model & Battery Capacity/kWh & Max Range/mile & Power/horsepower & Charging Time \\
\hline \multirow{2}{*}{ Tesla Model 3 } & 50 & 156 & 258 & $9 \mathrm{~h}$ \\
& 75 & 234 & 258 & $9 \mathrm{~h}$ \\
Tesla Model S & 40 & 125 & $360-370$ & $10 \mathrm{~h}$ \\
& 60 & 187 & $360-370$ & $10 \mathrm{~h}$ \\
Chevrolet Bolt EV & 14.44 & 265 & $360-370$ & $10 \mathrm{~h}$ \\
\hline
\end{tabular}

Table 3. The basic data for common charging piles.

\begin{tabular}{cc}
\hline Level & Rated output Power/kW \\
\hline 1 & $7.4 \mathrm{~kW}$ \\
2 & $22 \mathrm{~kW}$ \\
DC & $120 \mathrm{~kW}$ \\
Tesla Super & $400 \mathrm{~kW}$ \\
\hline
\end{tabular}


To simplify the calculation of the model, it is considered that the rated output power of the charging pile is a time of $22 \mathrm{~kW}$, and the charging time is $T_{\text {car }}=10 / a$.

In most common circumstances, the rate of demand for charging the electronic vehicles is directly proportional to the distance of the trip. Longer trip means greater energy consumption, which result in increasing needs for recharge the battery. When the destination is relatively close, the energy consumption is low due to the short driving distance, so as the demand for charging. Another factor is the average time people stay in the site of destination. If they need to stay longer, the demand for charging will increase as well. Let $\gamma$ be the rate of demand for charging per car, $d$ the distance of the trip and $L$ the maximum range provided by the battery capacity of the car, then $\gamma=d / L$. In real world, $\gamma$ is a real-time parameter that can be more accurately estimated by collecting customer-related information and big data process.

Let's build the model for a certain environment, say a theater or a supermarket. Let the number of active people in a certain place be $N$, in which the proportion of the population driving electric vehicles be $\delta$, the power demand rate be $\gamma$, the average power of electric vehicles be $P_{c a r}$, and the average charging time per car be $T_{c a r}$, which is not only determined by the activity time required by people in the very place but also affected by the efficiency of the charging pile. People have to wait for $d / L=\gamma$ of the battery to be charged, which is the power for the car to complete the journey back. Therefore, the charging time is at least $\gamma T_{\text {car }}$. Let $P$ denote the rated output power of the charging pile, the total electric energy consumption of the electric vehicle in the given place is:

$$
Q_{c a r}=\gamma^{2} \delta N P_{c a r} T_{c a r}=\left(\frac{d}{L}\right)^{2} \delta N P_{c a r} \frac{22 \times 10}{P_{c a r}}=220\left(\frac{d}{L}\right)^{2} d \delta N
$$

According to Hypothesis 2.3, the demand for electric energy is not directly related to the rated output power of the charging pile. The variation in the rate of demand for electric energy is determined by all parameters in the model, which should be real-time varying by different time and different locations.

For places that provide on-site charging piles within our daily life circle, such as our home, office, schools, etc. can provide fixed charging piles, the determination of $\gamma$ is independent of $d$, yet related to $D$, which denotes the sum of trips that have been taken after the previous charging, and $\gamma=D / L$. At this time, the consumption of electric energy can be derived by substituting $\gamma=D / L$ in the formula (3.2.2).

\section{- Computer Simulation and Model Accuracy Analysis}

The proportion $\delta$ of electric vehicles in the proportion of automobiles is increasing, with the advancement of automobile technology, the maximum average driving distance $L$ is also increasing. Let the rate of change of $\delta$ be $v_{1}(t)$, and the rate of change of $L$ be $v_{2}(t)$, in fact $v_{1}(t), v_{2}(t)$ are not easy to derive. We consider the linear model of stable growth of sales, that is, the growth rate of $\delta$ is a constant, and the growth rate of $L$ is small, thus the average growth rate is simulated. When $N=20000, d=100$, and $\lambda_{1}, \cdots, \lambda_{6}$ are 
assigned to be $0.2,0.05,0.3,0.3,0.02$, and 0.13 , respectively. The initial distance of $L$ is 145.6 by simply calculation, which will have a small growth rate. Figure 4 shows the variation of electric energy with time when $\delta$ and $L$ grow at different rates under these parameters.

Since the value of $L$ is related to the ratio of the six types of cars in $\lambda_{1}, \cdots, \lambda_{6}$, it is also related to the technical level of automobile production, so it is basically a piece-wise function of time. $\delta$ changes according to the above rules, and the change of $L$ is divided into the three stages, for the destination location, the average distance traveled by the customer to the location is equivalent to a constant. Figure 5 shows the relationship between power demand and time when $L$

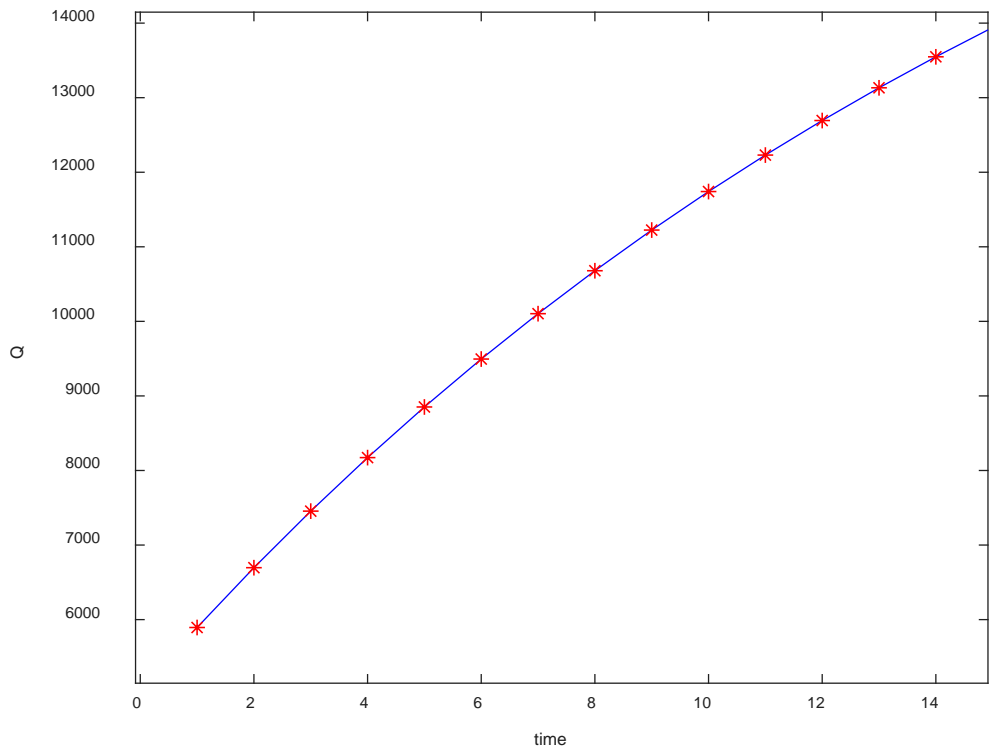

Figure 4. Power demand versus time ( $\delta$ and $L$ grow at different rates).

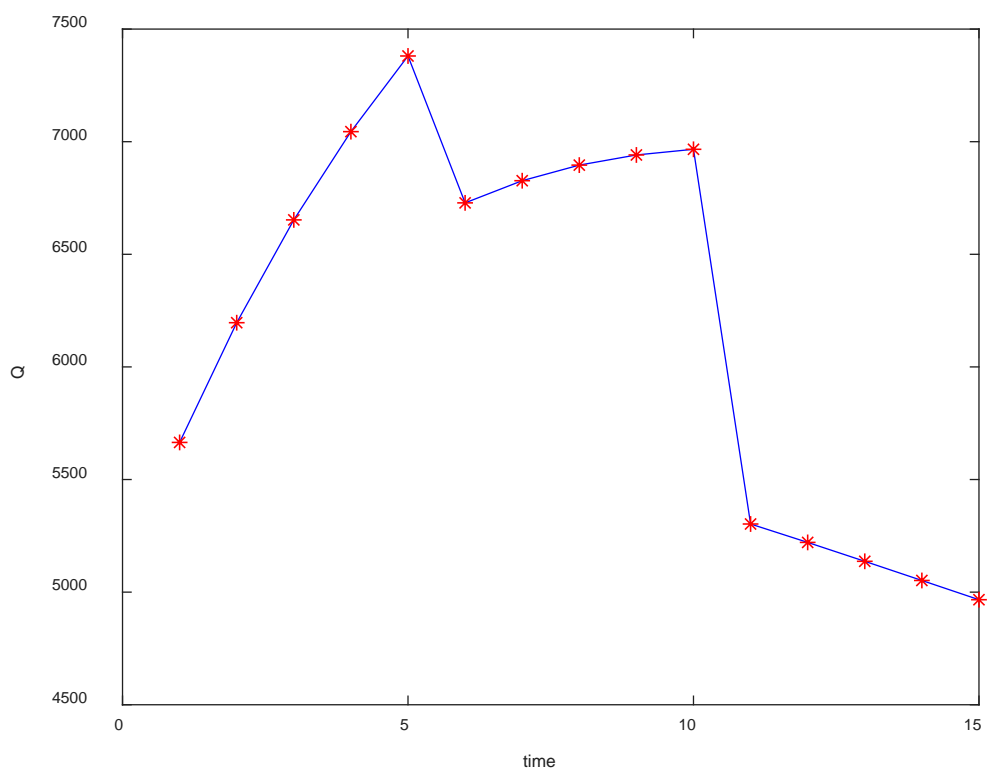

Figure 5. Power demand versus time (when $L$ jumps in three phases). 
jumps in three phases. As the performance of the car is getting better and better, the maximum driving distance is getting longer and longer, and the demand for charging in the middle of the journey is getting lower and lower, so the demand for electric energy in the place is gradually degraded. Although the population using electric vehicles is increasing, the number of people who need for charging has decreased, and the demand for electricity on the site has shown a downward trend. This decline in energy demand is only for this site, which means that as the car technology improves, people are more inclined to not charge the car in this place, but will choose to charge the car in other places, the energy consumption of other functional places will continue to increase. If the site wants customers to choose to charge here, it needs to be guided by policies, such as discounts on charging fees, subsidies, etc., to influence and increase the value of $\gamma$.

Different places mean different geographical locations. Let's choose sites with similar functions and different geographical locations, such as railway stations, airports, bus stations, etc. The average distance $d$ between customers and each site are different. Assuming the number of active people in the two sites is close, and when other parameters are kept unchanged, the power demand is a quadratic function of $d$, according to Figure 6 .

In fact, due to different locations and different functions, the customer groups coming to the site are different, so the proportion of the six models in different sites are not the same. Then the average maximum range $L$ is different. That is, $d$ and $L$ change simultaneously, Figure 7 gives the relationship between power demand and time when $L$ exhibits a phase change and $d$ continues to increase. This also coincides with the actual situation.

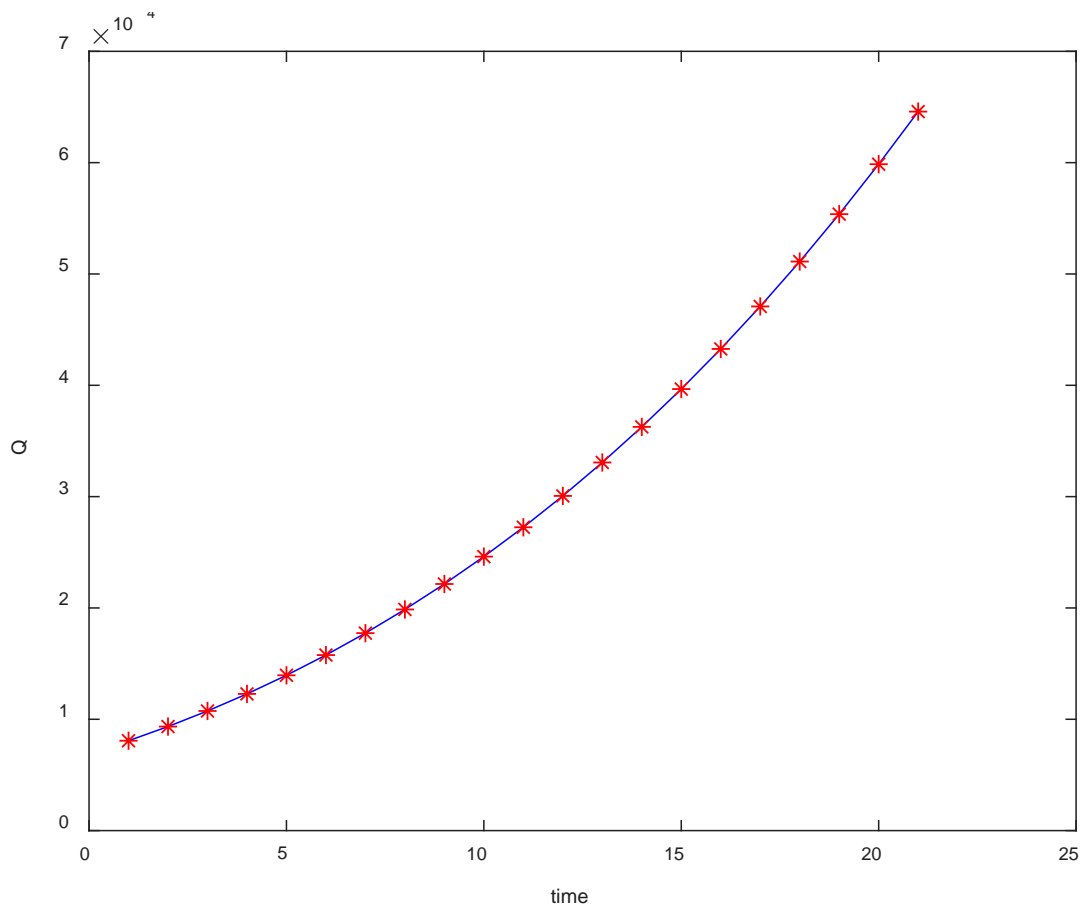

Figure 6. Power demand versus distance. 


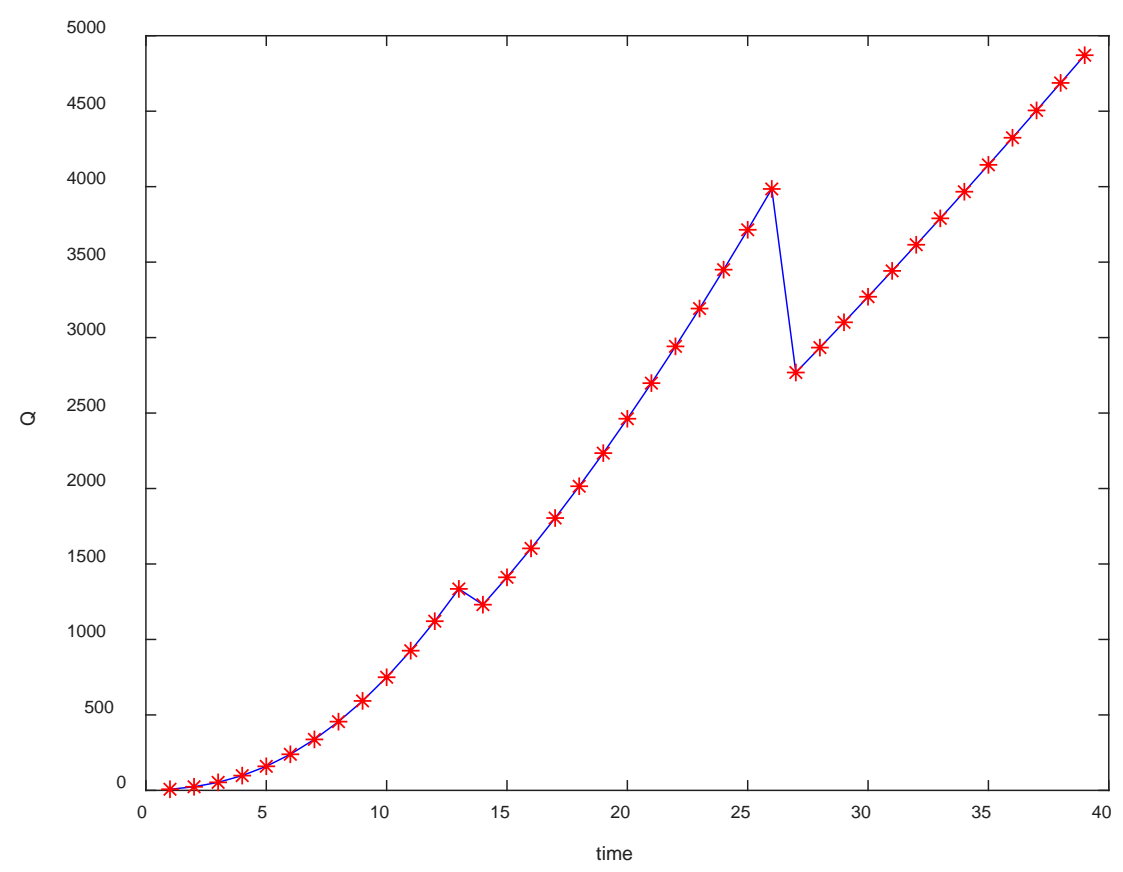

Figure 7. Power demand versus time.

\subsubsection{Increasing Demand for Power Exert Impact and Requirement to Public Sites}

\section{- The Impact and Requirements of Mobile Phone Charging on the Venue}

Mobile phones are almost always available, so the demand for charging is large, but the energy consumed by each mobile phone is relatively small. If an external charging socket is added in the place, the demand for the socket is relatively large, and the number of mobile phones connected to the circuit at the same time is also large, which is likely to cause a safety hazard. A portable mobile charging carrier can be considered to meet the demand. Simply install the equipment station with the carrier in a few fixed locations. Another advantage of this device is that people's activities will not be restricted by location. The number of mobile charging carriers is determined by the power demand calculated by the model and the carrier power. When the output power of the mobile carrier is relatively high, the charging time is short, the speed of the carrier circulation is fast, and the number can be reduced; but if the carrier output power is relatively low, the charging time is long, the carrier circulation slow flow rate is slow, and the quantity is much more needed.

\section{- The Impact and Requirements of Car Charging on the Site}

Electric vehicles need to be equipped with special charging piles, and they belong to high-power electric appliances. They have special requirements for the lines, and separate connection circuits must be established separately. The charging pile equipment required for a site is determined by the demand for electric energy of electric vehicles in the site, the preference for charging cost and time cost, and considering the broad prospects of electric vehicles, the share in the automotive market will increase day by day. This effect is delayed. 


\section{The Cost Model of Energy Growth}

In the previous chapter, we have studied the change in energy demand. In this section, we continue to study the law of cost change based on this increase in energy demand and the payment method.

\subsection{Problem Analysis}

In order to meet people's demand for electric energy, the site needs to add the matching charging facilities. The increased cost is mainly composed of three parts, namely, the cost of purchasing the matching charging facilities, the construction cost of reconstructing or adding the facilities, and the electricity consumption

\subsection{Establishment and Solution of Cost Model}

\subsubsection{Cost Model for Meeting the Electrical Requirements of Small Electronic Products}

According to the analysis in the previous section, we can know that if we want to add the cost model which meeting mobile phone power demand, the mobile charging carrier equipment station (or charging station with multiple outlets) should be the main parameter. Supposing the number of mobile carriers (or sockets) in a device station is $n$, The unit cost of the equipment station is $C_{1}$, the total number of mobile carriers should meet the customer demand with the proportion of $\mu$, then the number of equipment stations that need to be built in the site is:

$$
s=\left[\frac{\mu\left(N \delta_{1} \gamma_{1}+N \delta_{2} \gamma_{2}\right)}{n}\right],
$$

where $[x]$ is the integer part of $x, N$ is still indicating the population of the site; $\delta_{1}, \delta_{2}$ are representing the proportion of people carrying mobile phones and computers respectively; $\gamma_{1}, \gamma_{2}$ are representing the demand intensity for electricity from people using mobile phones and computers; $\mu$ denotes the proportion of meeting the energy needs of customers; Assuming the cost per kWh is $Q_{c}$; the average lifespan of each mobile charging carrier is $\omega$; then by the end of its life, the total cost of energy consumption is:

$$
C_{2}=\frac{\omega \mu Q_{c}}{1000},
$$

The construction cost of building an equipment station is $C_{3}$, so the total cost is

$$
C_{p}=S C_{1}+C_{2}+C_{3}=C_{1}\left[\frac{\mu\left(N \delta_{1} \gamma_{1}+N \delta_{2} \gamma_{2}\right)}{n}\right]+\frac{\omega \mu Q_{c}}{1000}+C_{3} .
$$

Assuming the average time for charging service at the site within one day are $\tau$ hours. Therefore, the average cost per hour per mobile charging carrier is:

$$
\overline{C_{p}}=\frac{C_{p}}{\omega \tau n S}
$$


$\overline{C_{p}}$ is the average cost of a mobile charging carrier (socket) within one hour. As a result that many parameters are estimated during the calculation, there is a certain error interval or range of variation. As far as one device station be concerned, $\mathrm{n}$ is different; the price is different, and the $S$ is different. At the same time, $S$ is in different quantity ranges and the discounts are different. That is, different unit prices result in different total costs. Taking these changes into consideration, a range of cost changes will be got. When customers rent for free, the cost is entirely borne by the merchant. Supposing the customer needs to pay a certain fee when renting these devices. If the customer pays more than $\overline{C_{p}}$, it means that the merchant makes money; If the customer pays less than $\overline{C_{p}}$, it means that the merchant has to bear a part of the cost; If the cost paid by the customer is $\overline{C_{p}}$, it means that this part of the cost is completely borne by the customer.

\subsubsection{Cost Model for Meeting the Electrical Requirements of Large Electronic Products}

Customers with high consumption levels are more likely to save time costs than economic costs; however, for the average users, the economic cost may be more important. Therefore, it should be distinguished according to the cost and performance of the charging pile when constructing one in a certain place.

It is assumed that three types of charging piles are installed in the site, the cost of Class A is lower, but the charging time is longer; the cost of Class B is increased, but the charging time is saved; Class $C$ is the highest cost and the charging time is the shortest. The above three types of charging piles are installed according to the proportion of the three types of customers on the site.

$N$ is assumed to be the number of active people in the venue; $\delta$ indicates the proportion of the population using electric vehicles; $\gamma$ denotes the demand intensity for electric energy using by electric vehicles; $\zeta_{A}, \zeta_{B}, \zeta_{C}$ represent the proportion of three types of customers among the population using electric vehicles. There is $\zeta_{A}+\zeta_{B}+\zeta_{C}=1 . C_{1 A}, C_{1 B}, C_{1 C}$ represent the unit price of three types of charging piles. In order to promote electric vehicles, many different preferential policies have been implemented, which may result in the same unit price of electric energy consumption for different types of charging piles. It is assumed that $c_{A}, c_{B}, c_{C}$ separately represent the unit price of electricity per kWh consumed using 3 types of charging piles. The proportion of meeting the needs of the three types of customers are $\mu_{A}, \mu_{B}, \mu_{C}$. Then the proportion of energy demand for the three types of customers are also $\mu_{A}, \mu_{B}, \mu_{C}$. As a result, the number of three types of charging piles that need to be built in the site is:

$$
S_{A}=\left[\mu_{A} \zeta_{A} N \delta \gamma\right], \quad S_{B}=\left[\mu_{B} \zeta_{B} N \delta \gamma\right], \quad S_{C}=\left[\mu_{C} \zeta_{C} N \delta \gamma\right] .
$$

It is assumed that the service life of three types of charging piles are $\omega_{A}, \omega_{B}$, $\omega_{C}$ respectively. Then, by the end of the life of the charging pile, the total costs of energy consumption provided are: 


$$
C_{2 A}=\frac{c_{A} \mu_{A} Q \omega_{A}}{1000}, \quad C_{2 B}=\frac{C_{B} \mu_{B} Q \omega_{B}}{1000}, \quad C_{2 C}=\frac{C_{C} \mu_{C} Q \omega_{C}}{1000} .
$$

The construction costs of constructing the three types of charging piles are $C_{3 A}, C_{3 B}, C_{3 C}$ respectively. So the total cost of these three types of charging piles are respectively:

The total cost of reconstructing to meet the site's energy needs is:

$$
\begin{aligned}
& C_{r A}=S_{A} C_{1 A}+C_{2 A}+C_{3 A}=C_{1 A}\left[\mu_{A} \zeta_{A} N \gamma\right]+\frac{C_{A} \mu_{A} \zeta_{A} Q \omega_{A}}{1000}+C_{3 A} \\
& C_{r B}=S_{B} C_{1 B}+C_{2 B}+C_{3 B}=C_{1 B}\left[\mu_{B} \zeta_{B} N \gamma\right]+\frac{C_{B} \mu_{B} \zeta_{B} Q \omega_{B}}{1000}+C_{3 B} \\
& C_{r C}=S_{C} C_{1 C}+C_{2 C}+C_{3 C}=C_{1 C}\left[\mu_{C} \zeta_{C} N \gamma\right]+\frac{C_{C} \mu_{C} \zeta_{C} Q \omega_{C}}{1000}+C_{3 C}
\end{aligned}
$$

The total cost of reconstructing to meet the site's energy needs is:

$$
C_{c a r}=C_{r A}+C_{r B}+C_{r C} \text {. }
$$

It is assumed that the average time for charging service at the site are $\tau$ hours within one day, so the average cost per hour for each type of charging pile in the three types of charging piles is:

$$
\overline{C_{r A}}=\frac{C_{r A}}{\omega \tau S_{A}}, \quad \overline{C_{r B}}=\frac{C_{r B}}{\omega \tau S_{B}}, \quad \overline{C_{r C}}=\frac{C_{r C}}{\omega \tau S_{C}} .
$$

$\zeta_{A}, \zeta_{B}, \zeta_{C}$ are the estimated parameters. It is difficult to estimate this parameter accurately because it changes in real-time in the actual situation. Therefore, the cost calculated according to the above formula will change within a certain range.

The average cost per hour for different types of charging piles is different. If the customer uses it for free, the cost is entirely borne by the merchant. If the customer pays less than the average cost per hour, it means that the merchant bears part of the cost. If the customer pays more than the average cost per hour, it means that the merchant makes money. If the customer pays an hourly fee equal to the average cost, it means that the cost is entirely borne by the customer.

\section{Establishment of Models for Different Environments}

In this section, we focus on four completely different public places. Based on the different use situations of small electronic products and large electronic products, the changes of specific model parameters and the adjustment of the model adjustments are given.

\subsection{Problem Analysis}

Through the research in the first two sections, the demand modes for energy and the demand for equipment in mobile phones and automobiles are different. Even for the same type of electronic products, there is a difference in the amount and manner of energy demand when the function of the activity places is differ- 
ent. This difference can be distinguished by the difference in parameters in the model. In this section, the differences in energy demand and the cost model between mobile phones and automobiles used in different locations are studied.

\subsection{School}

The school is characterized by a relatively fixed population and a long period of activity in the venue. Basically, everyone has mobile phones, computers and even more varieties of small electronic products. People can use the external patch panel to solve the charging problem, and the cost is basically solved by the user himself without an installation fee. Hence, the cost is mainly based on electricity. For cars, since the average activity time of people in the school is more than 8 hours, that is, the car needs to stop at a fixed place for about 8 hours, and there is no need to save the time cost of charging. Therefore, it is possible to select a charging pile with a low unit price and low power.

\subsubsection{Small Electronic Product}

The school is characterized by a relatively fixed population and a long period of activity in the venue. Basically, everyone has mobile phones, computers and even more varieties of small electronic products. People can use the external patch panel to solve the charging problem, and the cost is basically solved by the user himself without installation fee. Hence, the cost is mainly based on electricity. For cars, since the average activity time of people in the school is more than 8 hours, that is, the car needs to stop at a fixed place for about 8 hours, and there is no need to save the time cost of charging. Therefore, it is possible to select a charging pile with a low unit price and low power. And adapting (4.2.2)

$$
C_{p}=\frac{\omega \mu Q c}{1000} .
$$

\subsubsection{Large Electronic Product}

The active population is mainly composed of students and teachers, and the daily activities of the population are all around the school, generally no more than $10 \mathrm{~km}$, then $d=10$. Students are more inclined to use small electric vehicles. According to the formula (3), the value of $\lambda_{6}$ in the proportion of cars of type 6 is very high, much higher than the sum of the other five categories. Thus, the average maximum travel distance can be calculated. According to the model (4), people's demand for electric energy can be calculated. Since the population spends a lot of time on campus every day, the use of a Class B charging pile can meet the needs of people, so the total cost of energy consumption is calculated using formula (4.2.7):

$$
C_{r B}=S_{B} C_{1 B}+C_{2 B}+C_{3 B}=C_{1 B}\left[\mu_{B} \xi_{B} N \delta \gamma\right]+\frac{C_{B} \mu_{B} \xi_{B} Q \omega_{B}}{1000}+C_{3 B} .
$$

\subsection{Cafe}

The cafe is characterized by strong mobility of the active population, the number 
of people that can be accommodated is very limited, and the number is relatively low. The café with an area of $1000 \mathrm{~m}^{2}$ can generally accommodate about 150 200 people. People generally don't carry too many small electronic products, basically mobile phones. Of course, it is not excluded that some people like to study and work in cafes, so some people use computers. As a leisure place, the average time people spend in the cafe is relatively long, basically $2-4 \mathrm{~h}$. As a leisure place, the average time people spend in the cafe is relatively long and basically $2-4 \mathrm{~h}$. The number of parking spaces around the cafe is relatively small. From the perspective of cost, as more and more electric vehicles are used, cafes need to increase charging piles in order not to lose customers. People generally choose a cafe that is more convenient to travel, that is, the average distance to the destination is relatively small. At the same time, considering that people have a longer average residence time in the cafe, the B-type charging pile can fully meet the demand.

\subsubsection{Small Electronic Products}

According to the above analysis, the parameters in the model (2) can be set accordingly. When people are relaxing in cafes, the use rate of mobile phones is reduced, so the intensity $\gamma_{1}$ of demand for electrical energy using mobile phones. There are relatively few people who use computers to learn or work in cafes, so $\delta_{1}$ is close to 1 , and $\delta_{2}$ is very small. The intensity $\gamma_{2}$ of demand for electrical energy using a computer is significantly less than $\gamma_{1}$. The time for customers to use the computer in cafes is generally 2 - 4 hours, namely, in the

model, $\beta \in[1,2]$. Assume that, according to the general situation, the average mobile phone usage time $T$ of the customer is $5 \mathrm{~h}$, and the average time $T_{0}$ for continuous use of the mobile phone is $4 \mathrm{~h}$. According to the model (2), the average daily energy consumption in the site can be obtained.

When calculating the cost, the cafe is not designed to leave a lot of sockets in the wall, so the equipment that provides energy for mobile phones is mainly mobile charging equipment. The cost is composed of three parts, as the formula (4.2.3):

$$
C_{p}=S C_{1}+C_{2}+C_{3}=C_{1}\left[\frac{\mu\left(N \delta_{1} \gamma_{1}+N \delta_{2} \gamma_{2}\right)}{n}\right]+\frac{\omega \mu Q c}{1000}+C_{3} .
$$

\subsubsection{Large Electronic Equipment}

According to the above analysis, people tend to choose places that are close to home or company when they are leisurely. It is more likely to travel by a small electric car, and the average distance to the destination is smaller. Among the customers in this location, the value of $\lambda_{6}$ in the proportion of cars of type 6 is very high. The proportion parameters $\lambda_{1}, \cdots, \lambda_{6}$ of the six types of cars are different from the parameters in the school. According to this coefficient, the average maximum travel distance can be calculated, and according to the model (4), the demand of people for electric energy can be obtained.

Considering that there are fewer parking spaces around the cafe, the use of 
Class B charging piles can meet the needs of people, so the total cost of energy consumption expressed by formula (4.2.7):

$$
C_{r B}=S_{B} C_{1 B}+C_{2 B}+C_{3 B}=C_{1 B}\left[\mu_{B} \xi_{B} N \delta \gamma\right]+\frac{C_{B} \mu_{B} \xi_{B} Q \omega_{B}}{1000}+C_{3 B}
$$

The number of charging piles is formulated as follows

$$
S_{B}=[\gamma \mu M] \text {, }
$$

where $M$ represents the number of parking spaces provided by the cafe, which can meet the demand for the proportion $\mu$ of customers driving an electric car.

\subsection{Airport}

The airport is characterized by a very mobile population and a large population that can be accommodated. The daily passenger flow can exceed 10 million passengers a day, and some airports can reach more than 60 million. People usually arrive at the airport 1 hour before the flight departure. However, there are also cases where the flight is late. According to the hypothesis 5 , without consideration of the flight delay caused by special situations, and a large number of customers are stranded at the airport, the average delay time of the flight does not exceed $2 \mathrm{~h}$. In other words, the average time for a customer to stay at the airport is 3 hours. The airport is open daily from 6 am to $12 \mathrm{pm}$ for a total of 18 hours. The customer's stay at the airport is the airport opening hours. The retention time for a customer at the airport is one-sixth of the opening hours of the airport. Assuming that $F$ represents the passenger flow of the airport for one day, the approximate population in the airport can be approximated.

$$
N=\frac{1}{6} F .
$$

In order to better arrange the work of the airport, the number of flights departing every hour is roughly equal. As more and more people choose airplanes, without consideration of the difference between the number of passengers on different flights in different time periods, namely, there is little difference between the number of people at the airport during peak and trough periods.

\subsubsection{Small Electronic Products}

Due to travel, in order to ensure that the mobile phone is fully charged, the intensity $\gamma_{1}$ of demand for electrical energy using mobile phones is significantly increased. Meantime, many customers are accustomed to carrying mobile charging devices, so $\gamma_{1}$ are close to 1 or greater than 1 , but does not reach the level in school. Unless the work is in a hurry, few customers will still work when they are on standby at the airport. Therefore, customers who carry computers have a lower demand for electrical energy, $\gamma_{2}$ is relatively small, $\delta_{1}=1$ and $\delta_{2}$ is close to zero. Since the main way to spend time at the airport is to play the phone, the average time the customer uses the phone every day is extended, namely, $T>5.05 \mathrm{~h}$. What's more, limited by airport conditions, the average time for 
customers to use the computer does not exceed 3 hours, namely, $\beta=\frac{3}{5.05} \approx 0.59$. According to the model (2), the average electric energy consumed per day in this site can be obtained. Due to the large number of $N$, there is a great demand for charging sockets and mobile charging carriers, so the total cost is calculated by formula (4.2.3) as follows:

$$
C_{p}=S C_{1}+C_{2}+C_{3}=C_{1}\left[\frac{\mu\left(N \delta_{1} \gamma_{1}+N \delta_{2} \gamma_{2}\right)}{n}\right]+\frac{\omega \mu Q c}{1000}+C_{3} .
$$

\subsubsection{Large Electronic Equipment}

The airport is generally built in the suburbs, and the average distance to the destination is long. Many people choose to use public transport. With the continuous development of electric vehicles, more and more shuttle cars use electric vehicles. If the number of electric shuttles is sufficient, the B-type charging pile can be used for charging, but the charging time is long and the charging pile will be occupied for a long time. If the number of electric shuttles is relatively small, in order to ensure the smooth operation of the shuttle, it is required that the charging time should not be too long. This requires the installation of a C-type charging pile, which also saves space. Some customers have higher requirements for charging time, which requires a C-type charging pile. Some customers care more about the cost of charging and can accept B-type charging piles. Furthermore, some people stay at the airport for a long time, and the vehicles are parked for a long time, thus, they can accept A-type charging piles. The total cost is the sum of the charges of the three types of charging piles and can be calculated by formula (4.2.8) as follows.

$$
C_{c a r}=C_{r A}+C_{r B}+C_{r C}
$$

\subsection{Shopping Center}

It is characterized by strong personal fluidity. It has two distinct differences from the airport. Firstly, the flow of people changes with time and has peak and trough periods. Secondly, the total number of people flow in a day is far less than that of the airport.

In general, the flow of people $(F)$ in shopping malls is about 10,000 . We have to think about meeting the demand for electricity at peak times with a strength of $\mu$.

The shopping center is usually open from 9:00 to 21:00, namely $h=12$ hours. Suppose that the average time people spend in the shopping center is $T_{w}$. Then the average population in the shopping center is:

$$
N=\frac{T_{w}}{12} F
$$

Assuming that $\kappa$ is the ratio of the average number of people in the site at peak time and low time, then the average number of people in the site at a low time is: 


$$
N_{l}=\frac{2 N}{1+\kappa},
$$

The average number of people in the site at peak hours is:

$$
N_{u}=\frac{2 \kappa N}{1+\kappa} \text {. }
$$

\subsubsection{Small Electronic Products}

When people in the shopping center, the purpose of shopping and entertainment is obvious. During this period, the frequency of using mobile phones is relatively low. Customers who use mobile phones have a lower demand intensity $\left(\gamma_{1}\right)$ for electrical energy. There basically no existing customers who use computers. $\gamma_{2}$ can be considered as zero. According to the model (2), it is possible to calculate the average electric energy consumption at the peak of the population in this site every day.

$$
Q=\gamma_{1} \delta_{1} N_{u} t P=\gamma_{1} \delta_{1} \frac{N_{u} T t_{0} P}{T_{0}},
$$

Because people don't stay in one place for a long time, the charging equipment for customers is mainly based on mobile charging carriers. $n$ represents the number of mobile charging carriers included in one device station. Let's assume the duration of the population peak period is $T_{u}$ in one day, and the duration of the demographic trough is $T_{l}$. When calculating the cost, we modify $\tau$, that is:

$$
\tau^{\prime}=T_{u}+\frac{1}{\kappa} T_{l},
$$

Then the total cost is given by formula (4.2.3):

$$
C_{p}=S C_{1}+C_{2}+C_{3}=C_{1}\left[\frac{\mu N_{u} \delta \gamma_{1}}{n}\right]+\frac{\omega \mu Q c}{1000}+C_{3} .
$$

\subsubsection{Large Electronic Equipment}

People are accustomed to shopping centers that are closest to their place of residence or work. The average distance $d$ to the destination is short, and the demand $\gamma$ of people for electrical energy is also low. We still consider meeting the energy needs of customers with peak intensity $\mu$, so the daily demand for electricity in the site is calculated by formula (3.2.2) as follows.

$$
Q_{\text {car }}=220\left(\frac{d}{L}\right)^{2} \delta N_{u},
$$

The total cost of each type of charging pile is calculated respectively using formula (4.2.8), so the total cost is the sum of the three types of charging piles, that is:

$$
C_{c a r}=C_{r A}+C_{r B}+C_{r C}
$$

\section{Cost Optimization Model}

In Chapter 3 and Chapter 4, we studied the growth of energy and the generation 
of costs. This section focuses on how to reduce costs by determining optimal parameters and related measures without changing the requirements.

\subsection{Problem Analysis}

According to the cost model established in Section 5, when calculating the cost of energy growth caused by the use of mobile phones, the mobile charging carrier (or multi-sockets fixed charging device) is the main one. The number of mobile carriers stored on one device is different, the purchase cost of the entire device is different, and the greater the number of carriers, the higher the cost of the entire device, but it is not proportional to the number of carriers. In the case where the total number of demand is constant, the number of devices required is related to the number of carriers provided. The more mobile carriers, the fewer the number of devices required, and the less construction cost of the installation. When calculating the cost model generated by the electric vehicle using electric energy growth, the cost of different types of charging piles is different, the construction cost is different, and the determination of the quantity has a great influence on the total cost. Therefore, the optimal number of mobile charging carriers when the average cost per hour of a single charging device is minimized, and the optimal number of types of electric piles can be determined according to the model.

\subsection{Small Electronics Cost Optimization Models}

\subsubsection{Model Establishment}

When purchasing a product, the supplier often gives different unit prices depending on the quantity of the purchased product. This price is not linear with the quantity of the product.

In Chapter 5, we calculate the cost is:

$$
C_{p}=S C_{1}+C_{2}+C_{3}=C_{1}\left[\frac{\mu\left(N \delta_{1} \gamma_{1}+N \delta_{2} \gamma_{2}\right)}{n}\right]+\frac{\omega \mu Q c}{1000}+C_{3} .
$$

The total demand for mobile charging carriers is determined, that is $\mu\left(N \delta_{1} \gamma_{1}+N \delta_{2} \gamma_{2}\right)$, the larger $n$ is, the smaller $S$ is. When $n$ is different, that the unit price $C_{1}$ of the equipment station is different. $S$ is in different quantity ranges, $C_{1}$ enjoys different discounts. Since the construction of the equipment station is relatively simple, $C_{3}$ can be considered to be constant and has no effect on the cost $C_{2}$ of power consumption. Determining the appropriate $n$ can optimize the total cost without changing the requirements. So we can make it by determining the optimal $n$ value that is:

$$
C_{p}^{\prime}=S C_{1}=C_{1}\left[\frac{\mu\left(N \delta_{1} \gamma_{1}+N \delta_{2} \gamma_{2}\right)}{n}\right]
$$

which is in minimum size.

It is assumed that $n \in[a, b]$, for a device station, for each additional $n$, it is equivalent to adding one more mobile charging carrier. The cost of a mobile 
charging carrier is $z$, when $n \in\left[a_{1}, b_{1}\right]$, the fixed cost of the equipment $w$ station is unchanged, The unit price of the equipment station containing $\mathrm{n}$ mobile charging carriers is then $C=w+n z$. When $n \in\left[a_{1}, b_{1}\right]$, the fixed costs will change, Maybe set to $\eta w$, Then the unit price becomes $C=\eta w+n z$. When $S$ is in a different range, the price ratio between the unit prices is $\theta$. In different range intervals, the $\theta$ values are different. If you adjust $\mathrm{n}$, the interval of $\mathrm{S}$ changes, then there is:

$$
C_{1}=\left\{\begin{array}{l}
C, \quad S \in[l, h] \\
\theta C, \quad S \in\left[l^{\prime}, h^{\prime}\right]
\end{array}\right.
$$

In order to facilitate the calculation, the rounding function is approximately continuous, so there is:

$$
C_{p}^{\prime} \approx \begin{cases}(w+n z) \frac{\mu\left(N \gamma_{1}+N \gamma_{2}\right)}{n}, & \frac{\mu\left(N \gamma_{1}+N \gamma_{2}\right)}{n} \in[l, h], \\ \theta(w+n z) \frac{\mu\left(N \gamma_{1}+N \gamma_{2}\right)}{n}, & \frac{\mu\left(N \gamma_{1}+N \gamma_{2}\right)}{n} \in\left[l^{\prime}, h^{\prime}\right] .\end{cases}
$$

Let $\mu\left(N \delta_{1} \gamma_{1}+N \delta_{2} \gamma_{2}\right)=M$, then:

$$
C_{p}^{\prime} \approx\left\{\begin{array}{l}
\frac{w M}{n}+z M, \quad n \in\left[\frac{M}{l}, \frac{M}{h}\right], \\
\frac{\theta w M}{n}+\theta z M, \quad n \in\left[\frac{M}{l^{\prime}}, \frac{M}{h^{\prime}}\right] .
\end{array}\right.
$$

It can be seen that whether the cost price can be reduced is independent of the cost $z$ of a single mobile carrier, only related to $n$ and $S$, and related to the price ratio $\theta$ coefficient, and $\theta$ is related to $S$.

\subsubsection{Optimal Cost Calculation}

In order to better determine the relationship of $n$ and $\theta$, it is assumed that the total demand number $M=10,000$. If $S$ is less than 200 pieces, the original price will be reduced; if $S$ is between 200 - 400, the original price will be reduced by $15 \%$; if $S$ is between $400-600$, the original price will be reduced by $30 \%$; if $S$ is more than 600 , the original price will be reduced by $50 \%$. When each equipment station is equipped with 20 or less mobile charging carriers, the fixed cost is $w$; When equipped with 0 - 40 carriers, the fixed cost is 1.5 times of the original; when equipped with more than 40 carriers, the fixed cost is 2.5 times of the original; Up to 50 carriers are available, see Figure 8 for the relationship between cost $C_{p}^{\prime}$ and $n$. With this setting, each device station is equipped with 40 mobile charging carriers, which can minimize the total cost of the cost.

The above parameters can be adjusted according to the actual situation, and the value of $\mathrm{n}$ which makes the total cost the lowest are calculated according to the model.

\subsection{Large Electronics Cost Optimization Models}

According to the needs of different types of customers, three different types of 


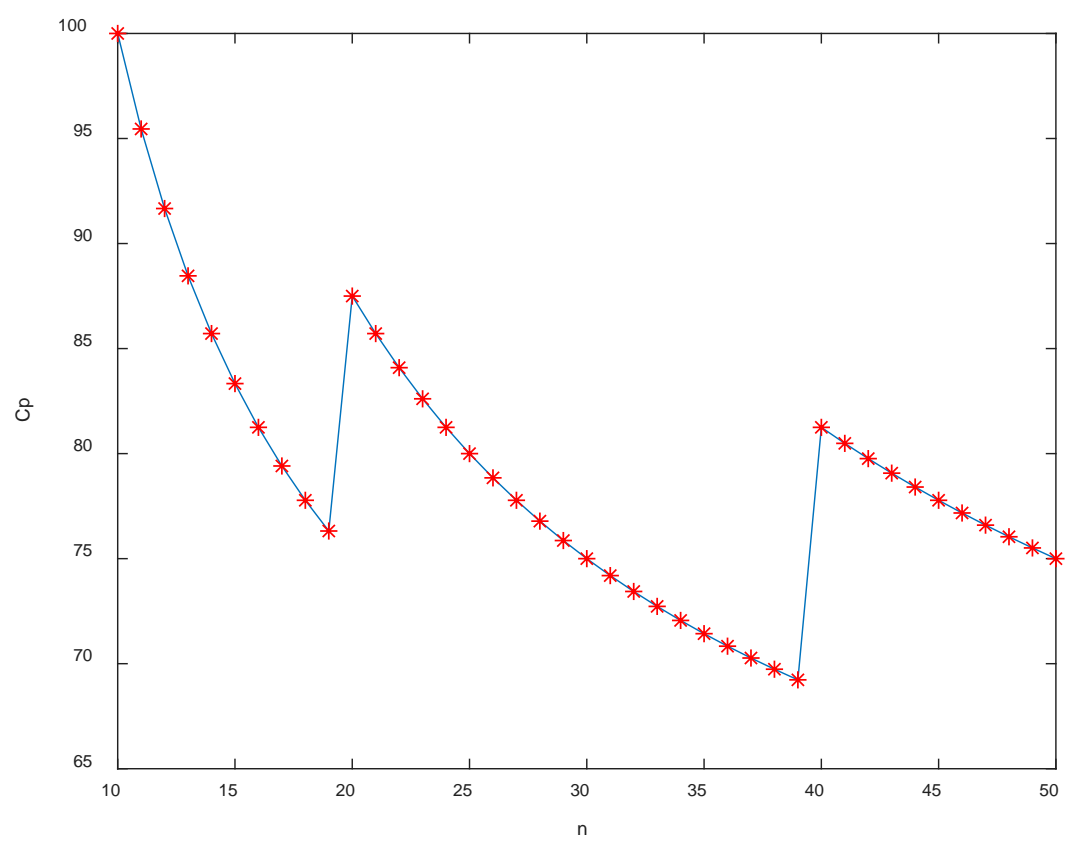

Figure 8. The relationship between cost $C_{p}^{\prime}$ and $n$.

car charging piles are installed on the site. According to Section 4, we know that the total cost of each type of charging pile is different. Regardless of the type of charging pile, the cost of a charging pile is relatively expensive. Therefore, the thinking when considering the optimization cost is different. Class A charging piles have low power and long charging time, which means that the average number of customers served during the one-day working hours is small and the cost is relatively low. Class $\mathrm{C}$ charging piles have high power and short charging time. The average number of customers in a day's working hours is large, and the number of customers who serve the same number of C-type charging piles is small, so the cost is low. However, customers may not choose this method because of the high cost of charging. Therefore, it can be considered to provide customers with a certain charging subsidy to encourage customers to choose a type $C$ charging pile, thereby reducing the number of charging piles installed. Since the requirements for the raw materials are different when the three charging piles are installed, the installation costs are different, but the difference is very small relative to the entire cost. In the cost optimization model, the impact of installation fee differences is not considered.

It is supposed that the number of customers who choose three types of charging piles A, B, C is respectively $N_{1}, N_{2}, N_{3}$, i.e. $\left[\mu_{A} \xi_{A} N \delta \gamma\right]=N_{1},\left[\mu_{B} \xi_{B} N \delta \gamma\right]=N_{2}$, $\left[\mu_{C} \xi_{C} N \delta \gamma\right]=N_{3}$, the power of class A charging pile is $22 \mathrm{KW}$, that of class $\mathrm{B}$ charging pile is $120 \mathrm{KW}$, and that of class $\mathrm{C}$ charging pile is $400 \mathrm{KW}$. During the period when A-class provides services for one customer, it can be seen that one B-class charging post provides services for six A-class customers, one C-class charging post can provide services for eighteen A-class customers and three B-class customers. The subsidy fee for a Class A customer who chooses a Class B 
charging pile is $C_{B}-C_{A}$, The subsidy fee for a Class A customer who chooses a Class $\mathrm{C}$ charging pile is $c_{C}-c_{A}$, The subsidy fee for a Class $\mathrm{B}$ customer who chooses $\mathrm{C}$ charging piles is $c_{C}-c_{B}$. Similarly, the original $\mathrm{B} \& \mathrm{C}$ customers will also receive the corresponding subsidies. The cost of setting up a Class A charging pile is $C_{A}$, it is possible to set the cost of the B-type charging pile to $C_{1 B}=k_{1} C_{1 A}$, The cost of the Class $C$ charging pile is $C_{1 C}=k_{2} C_{1 A}=k_{3} C_{1 B}$. Let the average energy required by each customer be $\sigma$, when the installation cost is not considered, the total cost of the original plan is:

$$
Z_{1}=N_{1}\left(C_{1 A}+c_{A} \sigma\right)+N_{2}\left(C_{2 B}+C_{B} \sigma\right)+N_{3}\left(C_{1 C}+C_{C} \sigma\right),
$$

Now set up $x_{1}, x_{2}, x_{3}, x_{4}, x_{5}$ represents the actual number of Class A charging piles respectively, in order to serve the actual number of Class $B$ charging piles required by Class $B$ customers, the number of Class $B$ charging piles required by Class A customers, the number of Class $\mathrm{C}$ charging piles required by Class $\mathrm{A}$ customers and the number of Class $\mathrm{C}$ charging piles required by Class $\mathrm{B}$ customers, so the total cost is:

$$
\begin{aligned}
Z_{2}= & x_{1} C_{1 A}+\left(x_{2}+x_{3}\right) C_{1 B}+\left(N_{3}+x_{4}+x_{5}\right) C_{1 C} \\
& +c_{A} \sigma x_{1}+\left(x_{2}+6 x_{3}\right)\left(c_{B}-c_{A}\right) \sigma+\left(N_{3}+18 x_{4}+3 x_{5}\right)\left(c_{C}-c_{A}\right)
\end{aligned}
$$

So the goal is to reduce the most cost, i.e.,

$$
\begin{aligned}
& \max \quad Z=Z_{1}-Z_{2}=\left(\left(N_{1}-x_{1}\right)+k_{1}\left(N_{2}-x_{2}-x_{3}\right)+k_{2}\left(x_{4}+x_{5}\right)\right) C_{1 A} \\
& +\left(N_{1}+N_{3}-x_{1}+x_{2}+6 x_{3}+18 x_{4}+x_{5}\right) c_{A}+\left(N_{2}-x_{2}-6 x_{3}\right) c_{B}-\left(18 x_{4}+3 x_{5}\right) c_{C}
\end{aligned}
$$

Needing to meet the needs of various types of customers, so there are constraints that are:

$$
\text { s.t. }\left\{\begin{array}{l}
x_{1}+6 x_{3}+18 x_{4} \geq N_{1}, \\
x_{2}+3 x_{5} \geq N_{2}, \\
x_{i} \geq 0, i=1, \cdots, 5
\end{array}\right.
$$

The optimal solution to the linear programming problem determined by the model (5) \& (6) is the most cost-effective optimization scheme in the case of meeting the demand.

\section{Model Evaluation}

The models developed in this paper are applicable to a wide range of applications and are suitable for a wide range of applications. For places with large differences in functions and properties, they can be distinguished and adjusted by the difference in parameter values in the model. Based on the idea of average estimation, the energy demand of the site is estimated, and the demand and quantity of equipment are determined according to the energy demand. At the same time, according to the idea of cost optimization, in the specific implementation process, the number of combinations of equipment is optimized to achieve the purpose of reducing costs. Analyze and model from the source of electricity and cost generation for easy implementation and calculation. At the same time, the 
change of the parameter value is used to reflect the change of the function of the place. Use a computer simulation to predict and estimate possible outcomes. At the same time, through the change law of parameters, the general law of electronic product development is simulated, and the law of power demand changes with time is given by computer simulation, which is convenient for predicting various possible changes. From the prediction results, it is in line with the actual development law.

However, since a large number of parameters in the model are estimated, the difference between the estimated values of some parameters and the actual value is not obvious. For example, the estimated rated power of the mobile phone charging has little effect on the results. In the application of the model, some data are more difficult to obtain accurate estimates. For example, we have selected six types of electric vehicles according to the market share. Other cars can be roughly divided into six types of electric vehicles according to the parameters. It is difficult to collect relatively accurate values for the proportion of the six types $\lambda_{1}, \cdots, \lambda_{6}$ of cars, so that there is a significant error in calculating the average maximum travel distance $L$. The effect of this parameter on the model is very obvious. Therefore, in practical applications, if there is an error in the collected data, there will be a certain deviation in the estimated value, which will have some impact on the result.

\section{Conclusions}

This paper obtains the following findings while modeling is adopted to research. In terms of charging services for small electronic products, when requirement quantity from customers is 10,000 , each equipment station is equipped with 40 mobile charging carriers, which can minimize the total costs. In terms of charging services for large electronic products, on the premise that all kinds of customers' charging requirements can be met, the total costs can be reduced by taking measures such as increasing the number of high-efficiency charging piles, reducing the number of low-efficiency charging piles, and subsidizing the selection and use of high-efficiency charging piles.

To effectively reduce the costs of charging in public places, this paper has the following suggestions. First of all, Public Places Charging Governance Committee, including delegates of government, enterprises and citizens, shall be established to mobilize all parties to promote a series of solutions. Secondly, government shall issue industry service guide to pilot reasonable layout of different types of public charging facilities. Finally, enterprises shall increase RD of high-efficiency charging piles so that the proportion of high-efficiency charging piles can be increased constantly.

Charging services in public places are basically free, but consumption of electronic energy will eventually be paid. This paper calls on everyone further to establish awareness of energy conservation and make a contribution to the construction of an environment-friendly society. 


\section{Conflicts of Interest}

The authors declare no conflicts of interest regarding the publication of this paper.

\section{References}

[1] Chen, G. (2017) Quality Control of Mobile Electronic Device Program. Information and Computer (Theoretical Edition), No. 8, 63.

[2] Xu, Y. (2019) Reflections on Building a Future Domestic Travel Service System. Auto Review, No. 12, 20.

[3] Qu, Y. (2013) Research on the Charging of Background Music in Public Places. People's Forum, No. 11, 144.

[4] http://detail.zol.com.cn/product_param/index2677.html

[5] http://baijiahao.baidu.com/s?id=1641268901105846105\&wfr=spider\&for=pc\&isFail $\underline{\text { Flag }=1}$

[6] https://zhuanlan.zhihu.com/p/28373625 


\section{Appendix}

Program 1 Calculate power consumption of mobile phone

clear, clc

$\mathrm{p}=[0.04,0.2,0.29,0.21,0.26]$;

time $=[0.5,2,4,6,10]$;

$\mathrm{T}=\mathrm{p}^{*}$ time';

$\% \mathrm{~T}=[5.44: 0.1: 7]$;

$\mathrm{N}=20,000$;

$\mathrm{r} 1=0.6, \mathrm{r} 2=0.5$;

$\mathrm{T} 0=4$;

$\mathrm{a}=65 / 15 ; \mathrm{b}=2$;

$\mathrm{P}=15$;

$\mathrm{d} 1=0.9 ; \mathrm{d} 2=0.4$

$\mathrm{t} 0=2$;

\section{\%\#\#\#\#\#\#\#\#\#\#\#\#\#\#\#\#\#\#\#\#\#\#\#\#\#}

$\% \mathrm{v}=0.03 ; \mathrm{b} 1=1.2 ; \mathrm{b} 2=1.4 ; \mathrm{b} 3=0.8 ; \mathrm{b} 4=0.6$;

$\% \mathrm{v} 1=\mathrm{b} 1^{\star} \mathrm{v} ; \mathrm{v} 2=\mathrm{b} 2^{*} \mathrm{v} ; \mathrm{v} 3=\mathrm{b} 3^{*} \mathrm{v} ; \mathrm{v} 4=\mathrm{b} 4^{*} \mathrm{v}$;

$\% \mathrm{~V} 1=0.3 ; \mathrm{V} 2=0.2$;

$\% \mathrm{LL}=[1: 30]$;

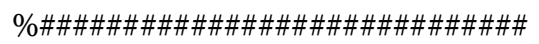

$\% \mathrm{TT}=\mathrm{LL} .{ }^{*} \mathrm{~V} 1+\mathrm{T}$;

$\% \operatorname{rr} 1=\mathrm{LL} .{ }^{*} \mathrm{v} 2+\mathrm{r} 1$;

$\% \operatorname{rr} 1=\operatorname{rr} 1 .{ }^{*}(\operatorname{rr} 1 \leq 1)+$ ones $(\operatorname{size}(\operatorname{rr} 1)) .{ }^{*}(\operatorname{rr} 1>1)$;

$\% \mathrm{dd} 2=\mathrm{d} 2-\left(\mathrm{LL} .{ }^{*} \mathrm{v} 3\right)$;

$\% \mathrm{dd} 2=\mathrm{dd} 2 .{ }^{*}(\mathrm{dd} 2 \geq \mathrm{V} 1)+\mathrm{V} 1^{*}$ ones $(\operatorname{size}(\mathrm{dd} 2)) .{ }^{*}(\mathrm{dd} 2<\mathrm{V} 1)$;

$\%$ rr2 $=$ r2-LL. ${ }^{*} \mathrm{v} 4$;

$\% \mathrm{rr} 2=\mathrm{rr} 2 .^{*}(\mathrm{rr} 2 \geq \mathrm{V} 2)+\mathrm{V} 2^{\star}$ ones $(\operatorname{size}(\mathrm{rr} 2)) .{ }^{*}(\mathrm{rr} 2<\mathrm{V} 2)$;

$\mathrm{rr} 1=\mathrm{r} 1 ; \mathrm{dd} 2=\mathrm{d} 2 ; \mathrm{rr} 2=\mathrm{r} 2 ; \mathrm{TT}=\mathrm{T}$;

$\mathrm{PP}=\mathrm{P}: 0.1: \mathrm{P}+3$;

$\mathrm{LL}=1$ : length $(\mathrm{PP})$;

\%\#\#\#\#\#\#\#\#\#\#\#\#\#\#

jieguo $=\left[\mathrm{TT}^{\prime}, \mathrm{rr1} 1^{\prime}, \mathrm{dd} 2^{\prime}, \mathrm{rr} 2^{\prime}\right]$

$\mathrm{Q}=\left(\mathrm{rr} 1 .{ }^{\star} \mathrm{d} 1+\mathrm{rr} 2 .{ }^{\star}\right.$ a. ${ }^{\star}$ b. $\left.{ }^{\star} \mathrm{dd} 2\right) .{ }^{\star} \mathrm{N} .{ }^{\star} \mathrm{TT} .{ }^{\star}$ to. ${ }^{\star} \mathrm{PP} . / \mathrm{T} 0$;

$\mathrm{Q} 1=\mathrm{Q} / 1000$;

$\operatorname{plot}\left(\mathrm{LL}, \mathrm{Q}, \mathrm{b}^{\prime}\right)$

hold on

$\operatorname{plot}\left(\mathrm{LL}, \mathrm{Q},{ }^{\prime \star} \mathrm{r}^{\prime}\right)$

xlabel('time')

ylabel('Q')

hold off

Program 2 Calculate power consumption of electric vehicle clear, clc

\%\#\#\#\#\#\#\#\#\#\#\#\#\#\#\# 


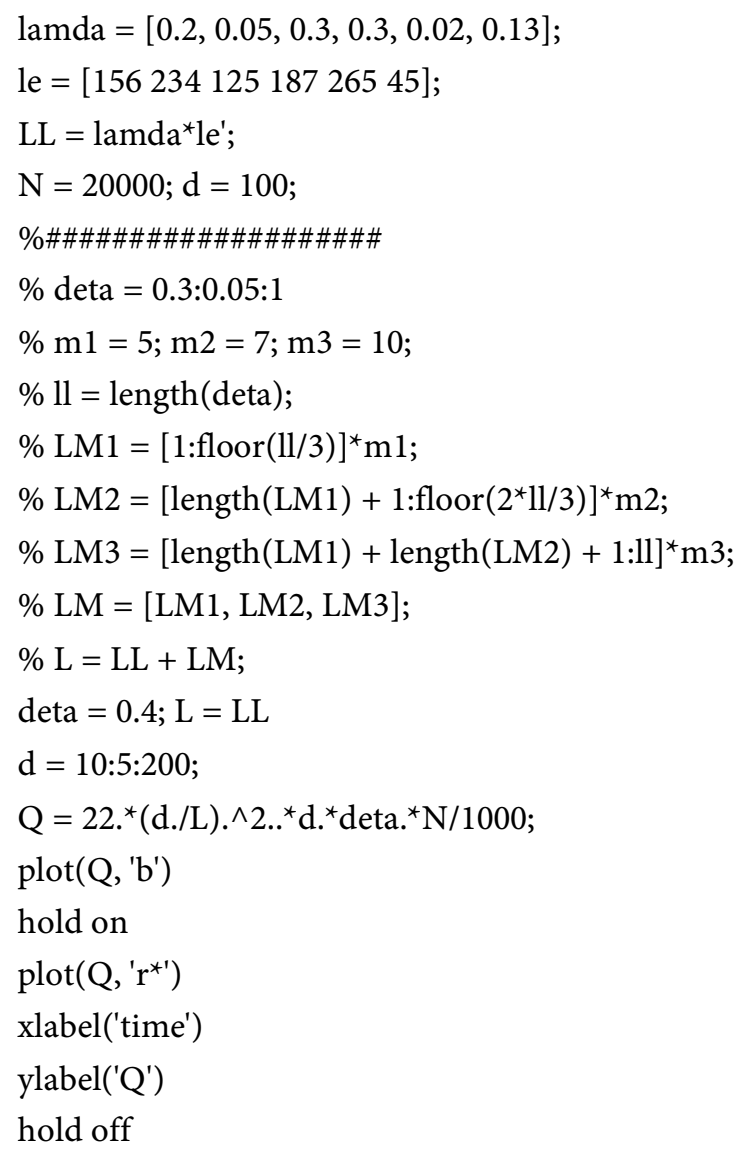

Program 3 The relationship between average demand intensity and coefficient $\mathrm{k} 3$

clear, clc

$\mathrm{M}=10000$;

$\mathrm{W}=100 ; \mathrm{z}=10$;

sn $=200$;

sita $=[1,0.85,0.7,0.5]$;

yita $=[1,1.5,2.5]$

$\mathrm{nn}=20$;

$\mathrm{N}=10: 1: 50$

for $\mathrm{p}=1$ :length $(\mathrm{N})$

$\mathrm{n}=\mathrm{N}(\mathrm{p})$;

$\mathrm{S}=$ floor $(\mathrm{M} / \mathrm{n})+1$;

te $=$ floor $(S / 50)$;

if te $\leq 4$

sita 1 = sita $($ te $)$;

else

$\operatorname{sita} 1=\operatorname{sita}(4)$;

end

$\mathrm{tn}=$ floor $(\mathrm{n} / \mathrm{nn})+1$

if $\mathrm{tn} \leq 3$ 


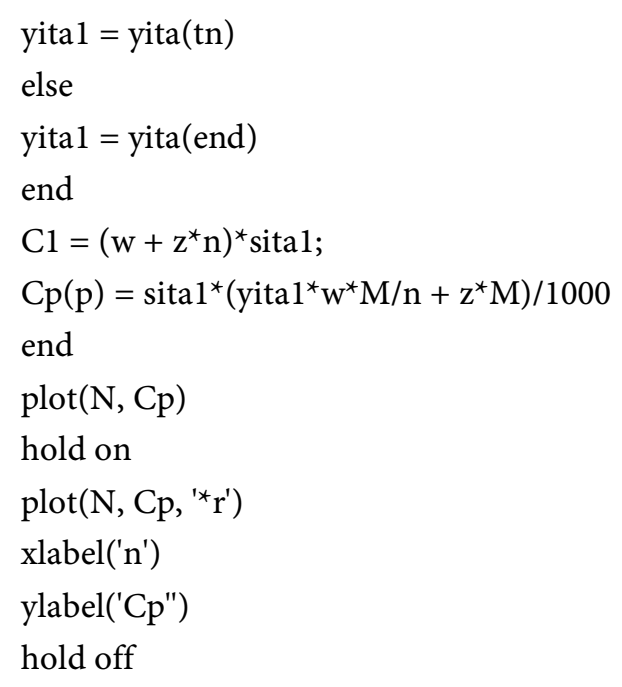

